\title{
Lattice Boltzmann method for incompressible flows with large pressure gradients
}

\author{
Yong Shi, T. S. Zhao,* and Z. L. Guo \\ Department of Mechanical Engineering, The Hong Kong University of Science and Technology, Clear Water Bay, \\ Kowloon, Hong Kong, China
}

(Received 10 March 2005; revised manuscript received 2 November 2005; published 15 February 2006)

\begin{abstract}
Conventional lattice Boltzmann Bhatnagar-Gross-Krook (LBGK) models can simulate incompressible flows correctly only if the Mach number $M$ and the density variation $\delta \rho$ are negligibly small. However, the equation of state $p=R T \rho$ resulting from the conventional models limits their application to incompressible flows with a rather small pressure gradient. In this paper, based on the Enskog equation, we propose a finite difference lattice BGK model for isothermal incompressible flows with the resulting equation of state and transport properties suitable for nonideal fluids. We validated this model by simulating the plane Poiseuille flow, the two dimensional Womersley flow, and the backward-facing step flow and found that the numerical results obtained by the proposed model are more accurate than those by the conventional LBGK models when the pressure gradient imposed on the flows increases.
\end{abstract}

DOI: $10.1103 /$ PhysRevE.73.026704

PACS number(s): 47.11.-j

\section{INTRODUCTION}

The lattice Boltzmann Bhatnagar-Gross-Krook (LBGK) method has been rapidly developed as a promising numerical algorithm for computation fluid dynamics over the last decade [1-5]. Among its various applications, it has been successfully applied to simulation of incompressible flows. However, the LBGK method virtually solves the compressible Navier-Stokes equations in the incompressible limit. Therefore an accurate simulation of incompressible flows using the LBGK method requires that the Mach number $M$ and the density variation $\delta \rho$ are negligibly small. Any deviation from these two requirements will lead to a so-called compressibility error $[6,7]$. Unfortunately, the equation of state resulting from conventional LBGK models, e.g., the D2Q9 model [17], is that of ideal gas, which implies that when conventional LBGK models are applied to simulation of incompressible flows at a given discrete particle speed $c$, the compressibility error, caused by density variation, will increase with increasing the pressure gradient. Therefore the conventional LBGK models are limited to simulation of isothermal incompressible flows with a rather small pressure gradient. This basic feature excludes the conventional LBGK models from simulating many practical engineering applications, such as the cooling system and flow through porous media, in which fluids are usually driven by a large pressure gradient.

Extensive efforts have been made to reduce or eliminate this compressibility error in simulations of incompressible flows with large pressure gradients. Two different approaches for modifying conventional LBGK models have been reported in the literature: the pressure models [6-10] and the density models $[11,12]$. In the pressure models, the fluid density is of little interest and not calculated in simulations. The macroscopic velocity is usually redefined based on a new "pressure" distribution function and the corresponding equi-

\footnotetext{
*Corresponding author. Electronic address: metzhao@ust.hk
}

librium distribution is appropriately constructed. The pressure models can recover the incompressible Navier-Stokes equations, but they are unsuitable for the multiphase or multicomponent flows and reactive flows, since in these flows the evolution of the density plays a significant role [12]. In the the density models, in which the density is still retained as an output variable in simulations. To suppress the compressibility error, in these models various treatments were introduced to modify the equation of state: for example, a feedback scheme was proposed [11]; a modified BGK model incorporating the repulsive interactions between particles was constructed [12].

As discussed earlier, the failure of the conventional LBGK models for incompressible flows with large pressure gradients is because the resulting equation of state is that of ideal gas, which causes the compressibility error to be increased with the pressure gradient. Therefore one of the most straightforward solutions to this problem is to develop an LBGK model for nonideal fluids. In line of this idea, we develop an Enskog-equation based LBGK (ELBGK) model in this work. In comparison with conventional LBGK models, this proposed ELBGK model takes the finite size of particles into account, resulting in the equation of state of nonideal fluids. Moreover, the model includes a term in its evolution equation to describe the nonlocal collision transfer resulting from the Enskog collision integral, thereby the transport properties derived from the model retain the same as those in the original Enskog theory [13-15]. We apply the general propagation scheme [16] to discretize the proposed ELBGK model and show that the resulting numerical scheme can be applied to simulation of large pressure gradient incompressible flows with the compressibility error controlled within an acceptable range.

The rest of the paper is organized as follows: In Sec. II, we briefly review on the conventional LBGK models with the equation of state of ideal gas. In Sec. III, an Enskog equation-based BGK model and the corresponding finite difference lattice version are presented. In Sec. IV, we apply the finite difference ELBGK model to simulate the two dimensional steady Poiseuille flow, the unsteady Wormersley flow, 
and the backward facing step flow. Finally some conclusions are drawn in Sec. V.

\section{LATTICE BGK MODEL BASED ON THE BOLTZMANN EQUATION}

In this section, we outline the conventional LBGK models with the equation of state of ideal gas. Without losing generality, we take the two dimensional nine-bit (D2Q9) model
[17] as an example, in which, the evolution equation reads

$$
f_{i}\left(t+\Delta t, \vec{r}+\vec{c}_{i} \Delta t\right)-f_{i}(t, \vec{r})=-\omega\left[f_{i}(t, \vec{r})-f_{i}^{e q}(t, \vec{r})\right],
$$

where $f_{i}$ is the single particle distribution function in the $i$ th particle velocity direction, and $t, \Delta t$, and $\vec{r}$ are the time, the time step, and the particle position, respectively. The dimensionless relaxation frequency $\omega=\Delta t / \tau$, with $\tau$ representing the relaxation time. The discrete velocities $\vec{c}_{i}$ are given by [17]

$$
\vec{c}_{i}= \begin{cases}(0,0), & i=0, \\ c\{\cos [(i-1) \pi / 2], \sin [(i-1) \pi / 2]\}, & i=1,2,3,4, \\ \sqrt{2} c\{\cos [(2 i-9) \pi / 4], \sin [(2 i-9) \pi / 4]\}, & i=5,6,7,8,\end{cases}
$$

where the particle speed $c=\sqrt{3 R T_{0}}$, with $T_{0}$ representing the reference temperature. In Eq. (1), $f_{i}^{e q}$ is the local equilibrium distribution function, which is given in a polynomial form:

$$
f_{i}^{e q}(t, \vec{r})=w_{i} \rho\left[1+\frac{\left(\vec{c}_{i} \cdot \vec{u}\right)}{\left(c_{s}^{i d}\right)^{2}}+\frac{\left(\vec{c}_{i} \cdot \vec{u}\right)^{2}}{2\left(c_{s}^{i d}\right)^{4}}-\frac{\vec{u}^{2}}{2\left(c_{s}^{i d}\right)^{2}}\right],
$$

where the sound speed $c_{s}^{i d}=\sqrt{3} c / 3=\sqrt{R T_{0}}$, the weight coefficients $w_{0}=4 / 9 ; w_{i}=1 / 9$, for $i=1,2,3,4$; and $w_{i}=1 / 36$, for $i=5,6,7,8 . \rho, \vec{u}$ are the density and macroscopic velocity. They are defined as

$$
\rho=\sum_{i=0}^{8} f_{i}=\sum_{i=0}^{8} f_{i}^{e q}, \quad \rho \vec{u}=\sum_{i=0}^{8} f_{i} \vec{c}_{i}=\sum_{i=0}^{8} f_{i}^{e q} \vec{c}_{i} .
$$

It should be noted that the discrete-velocity equilibrium distribution function $f_{i}^{e q}$, given by Eq. (3), is the second order Taylor expansion of the continuous Maxwell-Boltzmann distribution $[18,19]$. It is obtained by neglecting the terms in order of $O\left(\mathrm{M}^{3}\right)$ and beyond. Therefore for the LBGK models involving such a polynomial equilibrium distribution function, the Mach number under consideration should be small, i.e., $M \ll 1$.

With Eq. (4), Eqs. (1) and (3) can recover the macroscopic conservation like equations through the ChapmanEnskog procedure as

$$
\begin{gathered}
\frac{\partial u_{\beta}}{\partial x_{\beta}}+F^{I}=0 \\
\frac{\partial u_{\alpha}}{\partial t}+u_{\beta} \frac{\partial u_{\alpha}}{\partial x_{\beta}}=-\frac{1}{\rho} \frac{\partial p}{\partial x_{\alpha}}+\nu \frac{\partial}{\partial x_{\beta}}\left(\frac{\partial u_{\alpha}}{\partial x_{\beta}}\right)+\left(-\nu \frac{\partial F^{I}}{\partial x_{\alpha}}\right)+F^{I I}
\end{gathered}
$$

where

$$
F^{I}=\frac{1}{\rho}\left(\frac{\partial \rho}{\partial t}+u_{\beta} \frac{\partial \rho}{\partial x_{\beta}}\right)
$$

$$
F^{I I}=\frac{\nu}{\rho}\left\{\frac{\partial \rho}{\partial x_{\beta}} \frac{\partial u_{\alpha}}{\partial x_{\beta}}+\frac{\partial \rho}{\partial x_{\beta}} \frac{\partial u_{\beta}}{\partial x_{\alpha}}-\frac{\partial}{\partial x_{\beta}}\left[\frac{\partial}{\partial x_{\gamma}}\left(\frac{\rho u_{\alpha} u_{\beta} u_{\gamma}}{c_{s}^{2}}\right)\right]\right\},
$$

the kinematic viscosity $\nu=(1 / \omega-0.5)\left(c_{s}^{i d}\right)^{2} \Delta t$ and the equation of state is

$$
p=\rho\left(c_{s}^{i d}\right)^{2}=R T_{0} \rho .
$$

Equations (5) and (6) will reduce to the incompressible Navier-Stokes equations when the two additional terms $F^{I}$ and $F^{I I}$ vanish. This requires that the Mach number and the temporal and spatial variation of fluid density are small. Moreover, it is noted that the equation of state resulting from this conventional LBGK model is that of idea gas, from which the density variation with pressure can be given as

$$
\frac{\delta \rho}{\delta p}=1 /\left(c_{s}^{i d}\right)^{2}=3 / c^{2}
$$

Since in practical numerical simulations, the particle speed $c=\sqrt{3 R T_{0}} \approx 1$, Eq. (10) indicates that the fluid density variation is almost three times of the pressure variation. This means that the LBGK model given by Eqs. (1), (3), and (4) is limited to incompressible flows with a rather small pressure gradient.

\section{FINITE DIFFERENCE ENSKOG-EQUATION BASED LATTICE BGK MODEL}

The discussion in the preceding section shows that the reason the conventional LBGK models are limited to incompressible flows with a small pressure gradient is because of the equation of state of ideal gas. We now present a lattice BGK model based on the well recognized Enskog theory for nonideal fluids and show the resulting equation of state is that of nonideal fluids.

\section{A. Continuous Enskog equation-based BGK model}

The Enskog equation is given as [13] 


$$
\frac{D f}{D t}=\frac{\partial_{e} f}{\partial t},
$$

where $f$ is the singlet particle distribution function, $D / D t$ $=\partial / \partial t+\vec{c} \cdot(\partial / \partial \vec{r})$, with $t, \vec{c}$, and $\vec{r}$ representing the time, particle velocity, and particle position, respectively. In Eq. (11), the collision term $\partial_{e} f / \partial t$ is defined as [13]

$$
\begin{aligned}
\frac{\partial_{e} f}{\partial t}= & \iint\left[g\left(\vec{r}+\frac{1}{2} \sigma \vec{k}\right)\right. \\
& \left.\times f^{\prime}(\vec{r}) f_{1}^{\prime}(\vec{r}+\sigma \vec{k})-g\left(\vec{r}-\frac{1}{2} \sigma \vec{k}\right) f(\vec{r}) f_{1}(\vec{r}-\sigma \vec{k})\right] \sigma^{2}\left(\vec{c}_{1}\right. \\
& -\vec{c}) \cdot \vec{k} d \vec{k} d \vec{c}_{1},
\end{aligned}
$$

where $f^{\prime}(\vec{r}), f(\vec{r}), f_{1}^{\prime}(\vec{r}+\sigma \vec{k})$, and $f_{1}(\vec{r}-\sigma \vec{k})$ are the abbreviations of $f\left(\vec{r}, \vec{c}^{\prime}\right), f(\vec{r}, \vec{c}), f\left(\vec{r}+\sigma \vec{k}, \vec{c}_{1}^{\prime}\right)$, and $f\left(\vec{r}-\sigma \vec{k}, \vec{c}_{1}\right), \sigma$ is the diameter of particles, $g$ is the pair correlation function, and $\vec{k}$ is a unit vector pointing to the center of the coming collision particle of $f_{1}$ from the center of the test particle of $f$. The distribution function $f$ and pair correlation function $g$ in Eq. (12) can be expanded into a Taylor series about the local position $\vec{r}$ when the gas varies slowly in space. Neglecting the terms involving the second derivatives and beyond, the Enskog equation can be approximated as $[13,14]$

$$
\frac{D f}{D t}=J_{1}+J_{2}+J_{3}
$$

where

$$
\begin{gathered}
J_{1}=g \iint\left(f^{\prime} f_{1}^{\prime}-f f_{1}\right) \sigma^{2}\left(\vec{c}_{1}-\vec{c}\right) \cdot \vec{k} d \vec{k} d \overrightarrow{c_{1}}, \\
J_{2}=\sigma g \iint \vec{k} \cdot\left(f^{\prime} \frac{\partial f_{1}^{\prime}}{\partial \vec{r}}+f \frac{\partial f_{1}}{\partial \vec{r}}\right) \sigma^{2}\left(\vec{c}_{1}-\vec{c}\right) \cdot \vec{k} d \vec{k} d \vec{c}_{1}, \\
J_{3}=\frac{\sigma}{2} \iint\left(\vec{k} \cdot \frac{\partial g}{\partial \vec{r}}\right)\left(f^{\prime} f_{1}^{\prime}+f f_{1}\right) \sigma^{2}\left(\vec{c}_{1}-\vec{c}\right) \cdot \vec{k} d \vec{k} d \vec{c}_{1} .
\end{gathered}
$$

However, Eq. (13) cannot directly be applied to numerical simulations since the terms of $J_{1}, J_{2}$, and $J_{3}$, given by Eqs. (14)-(16), are rather complicated. Therefore we propose a simple BGK model to approximate Eq. (13), which reads

$$
\frac{\partial f}{\partial t}+\vec{c} \cdot \frac{\partial}{\partial \vec{r}} f=-\frac{g}{\tau^{\prime}}\left(f-f^{e q}\right)+J^{I}+J^{I I},
$$

where

$$
\begin{gathered}
\tau^{\prime}=\tau[1+2 b \rho g /(D+2)], \\
J^{I}=-f^{e q} b \rho g(\vec{c}-\vec{u}) \cdot \frac{\partial}{\partial \vec{r}} \ln \left(\rho^{2} g\right),
\end{gathered}
$$

$$
J^{I I}=-\frac{2}{D+2} \vec{c} \cdot \frac{\partial}{\partial \vec{r}}\left[b \rho g\left(f-f^{e q}\right)\right],
$$

$b=2 \pi \sigma^{3} /(3 m)$, and the local equilibrium distribution function $f^{e q}$ is given as

$$
f^{e q}=\rho / m(2 \pi R T)^{-D / 2} \exp \left[-(\vec{c}-\vec{u})^{2} / 2 R T\right],
$$

with $m, R$, and $D$ representing the mass of one particle, the gas constant, and the space dimension, respectively. Note that the BGK assumption, the first term on the right-hand side of Eq. (17), approximates the term of $J_{1}$ given by Eq. (14). It should also be mentioned that the contribution of $J_{2}$ and $J_{3}$ to the leading nonequilibrium expanded term of the distribution function, viz. $f^{(1)}$, is taken account by the new relaxation time $\tau^{\prime}$ given by Eq. (18). The term of $J^{I}$ is the first order approximation of $J_{2}$ and $J_{3}[20-22]$, while $J^{I I}$ describes the effect of the collision transfer of $J_{2}$ and $J_{3}$ on the transport properties. It can be demonstrated that for isothermal flows, through the Chapman-Enskog procedure, Eq. (17), along with Eq. (21), can recover the Navier-Stokes equations with the identical equation of state of nonideal fluid and the transport properties as those resulting from the Enskog equation.

\section{B. Finite difference Enskog equation-based LBGK model}

We now discretize the Enskog BGK model given by Eq. (17) over the particle velocity space $V(\vec{c})$, the physical space $\vec{r}$, and the time $t$ to obtain its corresponding finite difference lattice version. For simplicity, we focus on two dimensional problems, and extension to three dimensional is straightforward.

We first discretize the velocity space $V(\vec{c})$. By choosing the discrete particle velocity set $\left\{\vec{c}_{i}\right\}$ given by Eq. (2), one can write the corresponding discrete velocity equation as

$$
\frac{\partial f_{i}}{\partial t}+\vec{c}_{i} \cdot \frac{\partial}{\partial \vec{r}} f_{i}=-\frac{g}{\tau^{\prime}}\left[f_{i}(t, \vec{r})-f_{i}^{e q}(t, \vec{r})\right]+J_{i}^{I}+J_{i}^{I I},
$$

where

$$
\begin{gathered}
J_{i}^{I}=-f_{i}^{e q} b \rho g\left(\vec{c}_{i}-\vec{u}\right) \cdot \frac{\partial}{\partial \vec{r}} \ln \left(\rho^{2} g\right), \\
J_{i}^{I I}=-\frac{1}{2} \vec{c}_{i} \cdot \frac{\partial}{\partial \vec{r}}\left[b \rho g\left(f_{i}-f_{i}^{e q}\right)\right],
\end{gathered}
$$

and the discrete local equilibrium distribution function $f_{i}^{e q}$ is given by Eq. (3). The pair correlation function $g$ is expanded in terms of the density [15]:

$$
g=1+\frac{5}{8} b \rho+0.2869(b \rho)^{2}+0.1103(b \rho)^{3}+0.0386(b \rho)^{4},
$$

and the corresponding density $\rho$ and macroscopic velocity $\vec{u}$ are still defined by Eq. (4).

Then, we discretize the physical space $\vec{r}$ and the time $t$. In order to obtain a stable numerical algorithm, we use the general propagation scheme proposed by Guo et al. in Ref. [16] 


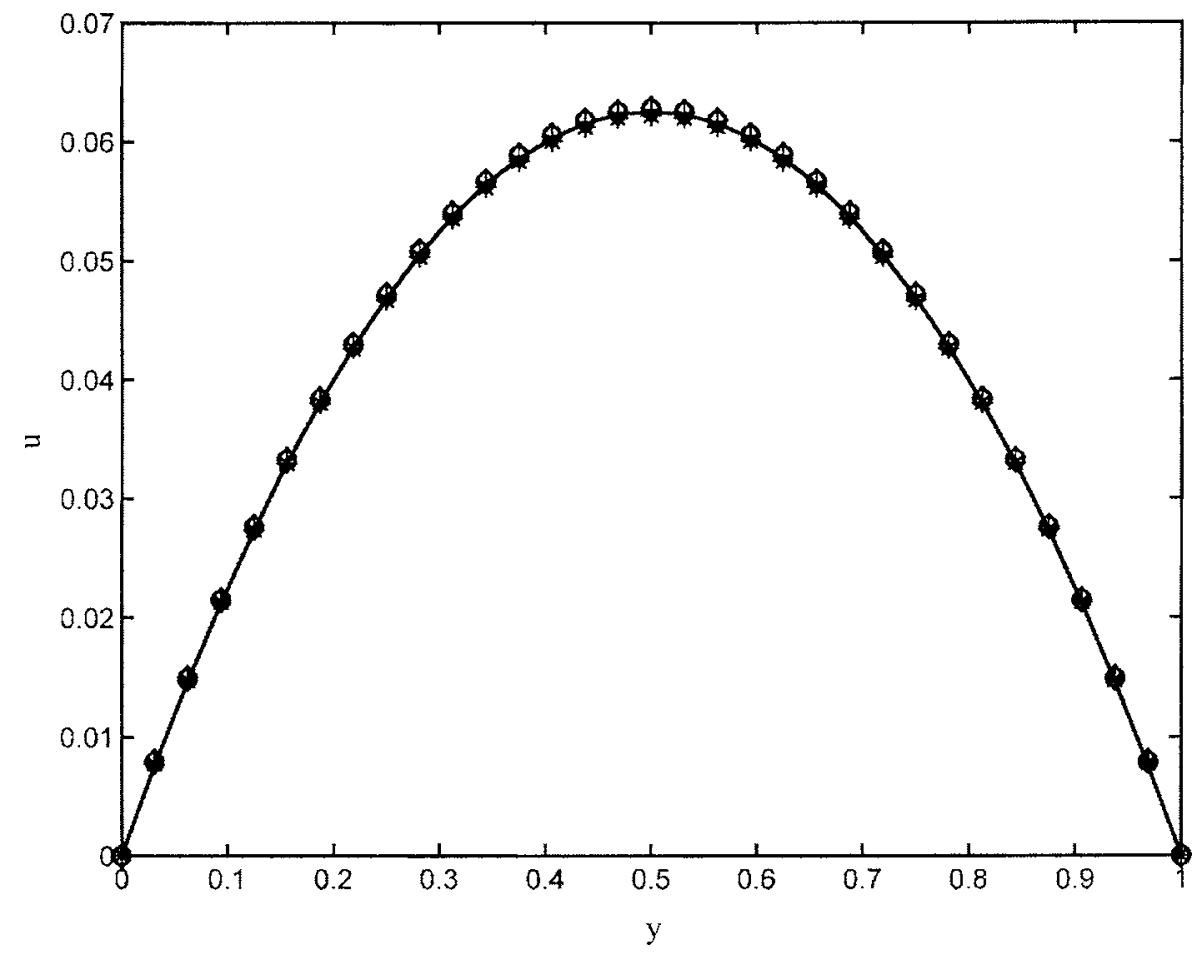

FIG. 1. The dimensionless horizontal velocity in Poiseuille flow across the channel at $d p / d x$ $=5.0 \times 1.0^{-3}$. Solid line: analytical solution; *: the conventional LBGK model; +: the ELBGK model with $b \rho=0.5$; $\diamond$ : by the ELBGK model with $b \rho=1.0 ; \bigcirc$ : the ELBGK model with $b \rho=1.5$.

to discretize Eq. (22). The resulting finite difference numerical algorithm consists of two basic formulations:

$$
h_{i}^{+}(t, \vec{r})=h_{i}(t, \vec{r})-g \omega^{\prime}\left[h_{i}(t, \vec{r})-h_{i}^{e q}(t, \vec{r})\right]+\Delta t\left(J_{i}^{I}+J_{i}^{I I}\right)
$$

and

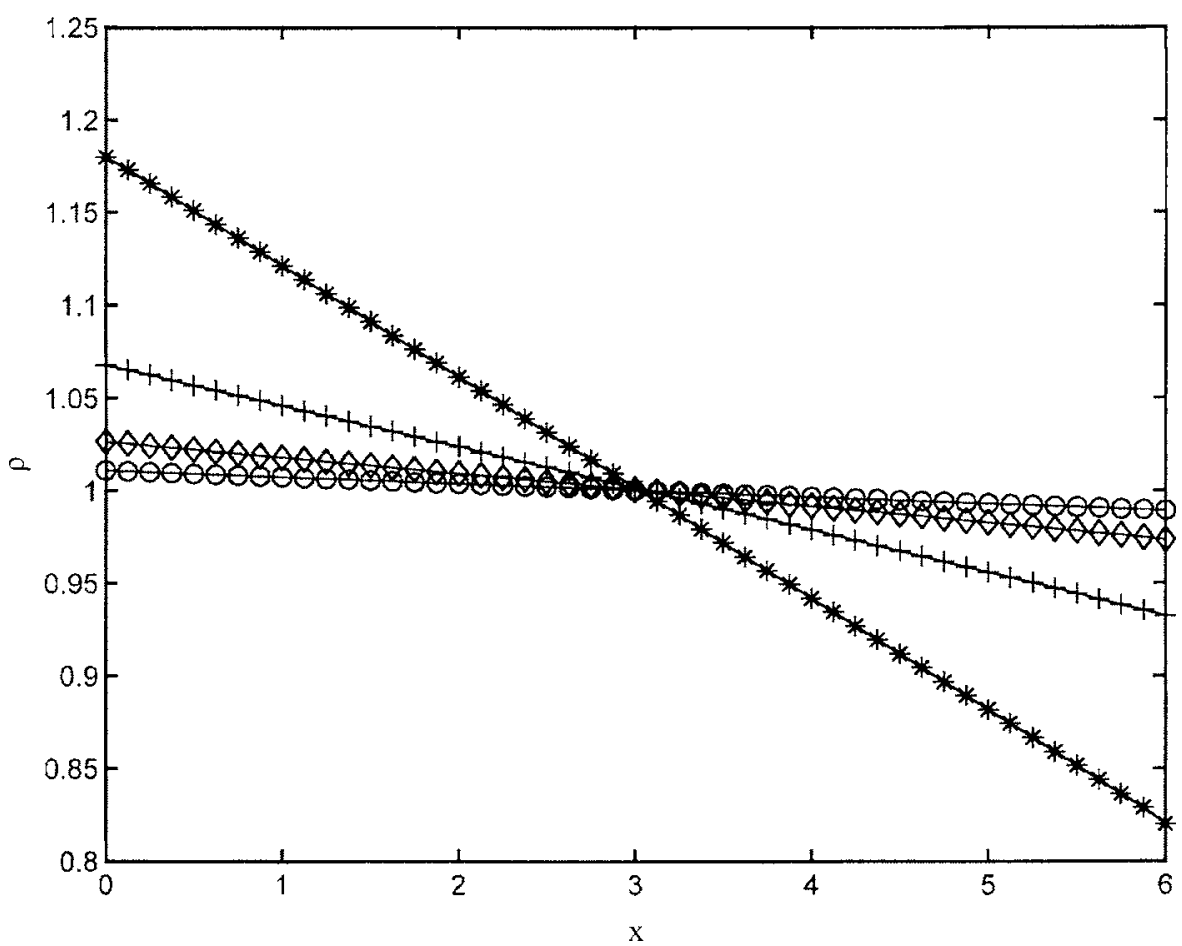

$$
\begin{aligned}
h_{i}(t+\Delta t, \vec{r})= & h_{i}^{+}(t, \vec{r})+\frac{d}{2}\left[h_{i}^{+}\left(t, \vec{r}+\vec{c}_{i} \Delta t / A\right)-2 h_{i}^{+}(t, \vec{r})\right. \\
& \left.+h_{i}^{+}\left(t, \vec{r}-\vec{c}_{i} \Delta t / A\right)\right]-\frac{A}{2}\left[h_{i}^{+}\left(t, \vec{r}+\vec{c}_{i} \Delta t / A\right)\right. \\
& \left.-h_{i}^{+}\left(t, \vec{r}-\vec{c}_{i} \Delta t / A\right)\right],
\end{aligned}
$$

where $\omega^{\prime}$ is the dimensionless relaxation frequency, equal to $\Delta t / \tau^{\prime}, A$ is the Courant-Friedricks-Lewey number, defined as
FIG. 2. The density variation along the channel in Poiseuille flow at $d p / d x=2.0 \times 1.0^{-2}$.*: the conventional LBGK model; +: the ELBGK model with $b \rho=0.5 ; \diamond$ : the ELBGK model with $b \rho=1.0$; $\bigcirc$ : the ELBGK model with $b \rho$ $=1.5$. 


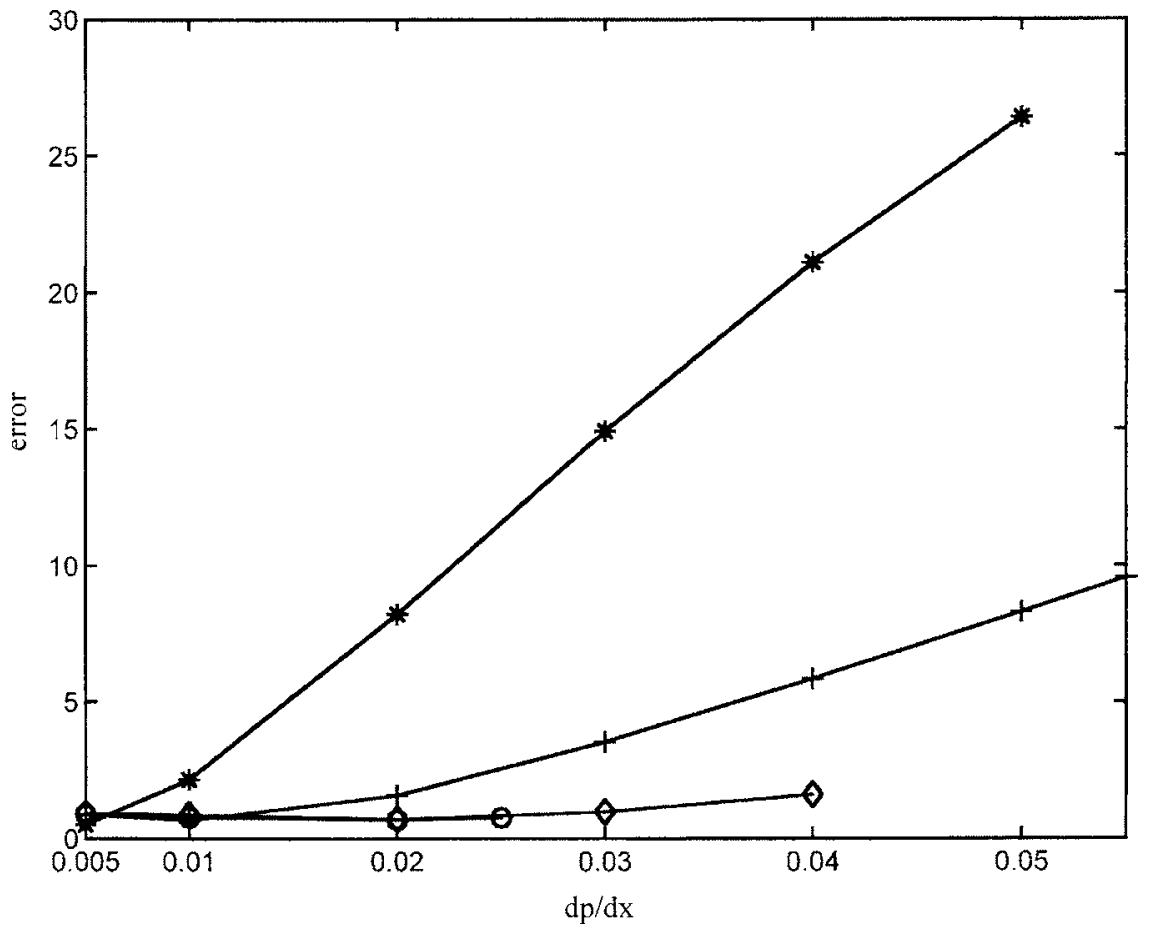

FIG. 3. The relative error of the center velocity in Poiseuille flow at different pressure gradient. -*: $_{-}$the conventional LBGK model; - +- : the ELBGK model with $b \rho=0.5 ;-\diamond-:$ the ELBGK model with $b \rho=1.0$; -O-: the ELBKG model with $b \rho=1.5$.

$A=c \Delta t / \Delta x$, with $\Delta x$ being the grid spacing. $h_{i}$ is a new distribution function, defined as [23]

$$
h_{i}=f_{i}-\Delta t J_{i}^{I} / 2
$$

and its equilibrium is

$$
h_{i}^{e q}=f_{i}^{e q}-\Delta t J_{i}^{I} / 2
$$

Equations (26) and (27) together describe the change of the distribution function at the position $\vec{r}$ in one time step $\Delta t$. Specifically, Eq. (26) describes the change of the distribution function due to the particle collision, while Eq. (27) describes the change due to particle streaming. In Eq. (27), we introduce a numerical parameter $d$. It can vary from $A^{2}$ to 1.0 [16]. Moreover, when $d=A^{2}$, Eq. (27) reduces to the socalled Lax-Wendroff scheme.

Now the density and macroscopic velocity can be defined in terms of $h_{i}$ as

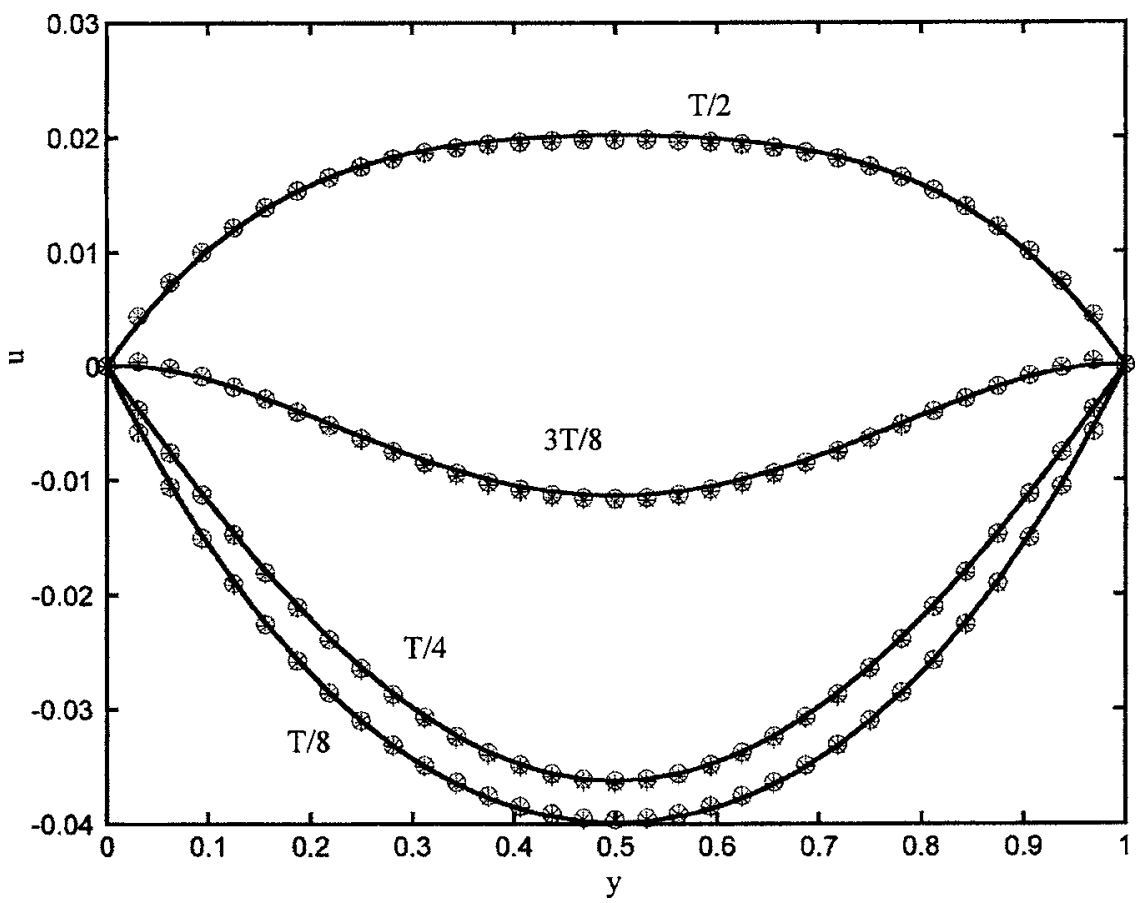

FIG. 4. The center velocity profile in Womersley flow with $d p d x=0.01 \cos (\omega t)$. Solid line: analytical solution; *: the conventional LBGK model; $\bigcirc$ : by the ELBGK model with $b \rho=1.5$. 


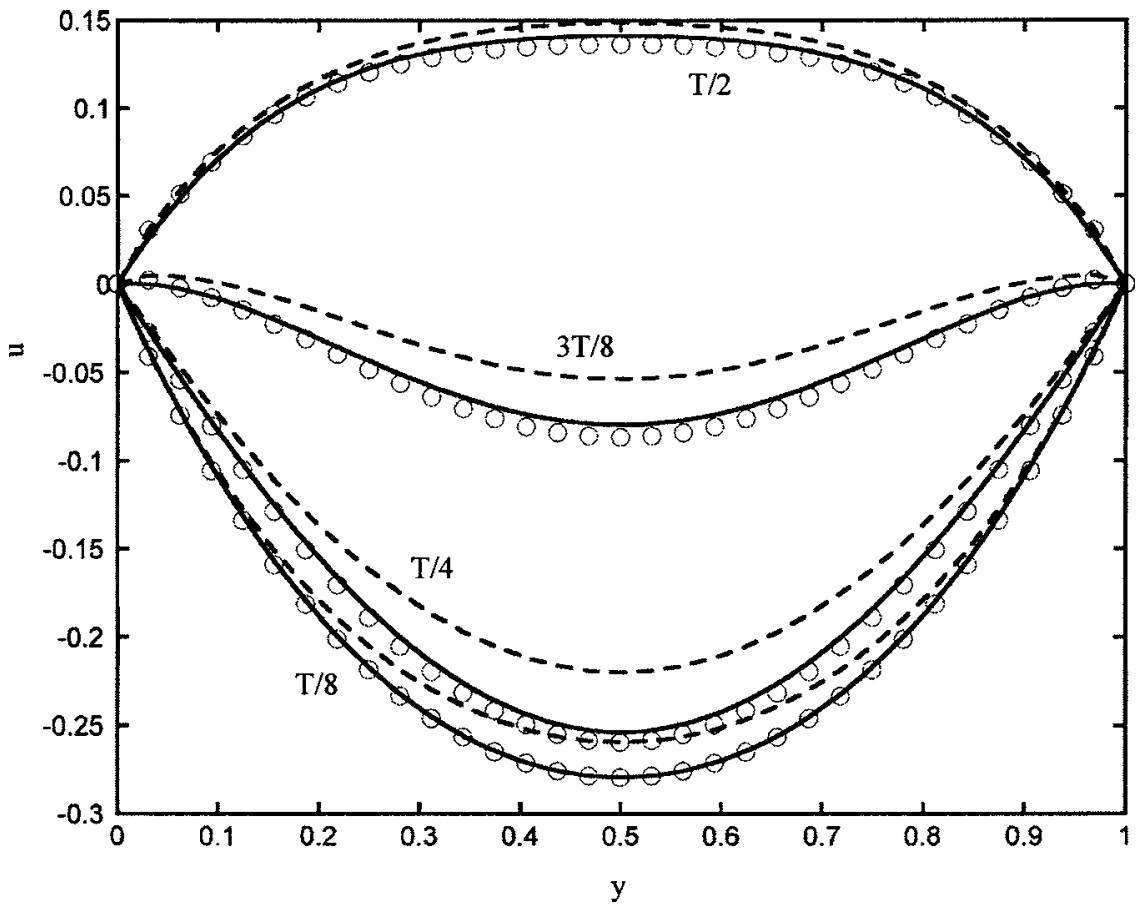

FIG. 5. The center velocity profile in Womersley flow with $d p / d x=0.07 \cos (\omega t)$. Solid line: analytical solution; dashed line: the conventional LBGK model; $\bigcirc$ : the ELBGK model with $b \rho=1.5$.

$$
\rho=\sum_{i} m h_{i}, \quad \rho \vec{u}=\sum_{i} m h_{i} \vec{c}_{i}-\frac{\Delta t}{2} b\left(c_{s}^{i d}\right)^{2} \frac{\partial}{\partial \vec{r}}\left(\rho^{2} g\right) .
$$

Through the Chapman-Enskog procedure, Eqs. (26), (27), and (30) can recover the equations of mass and momentum conservation (see the Appendix for details) with the viscosity $\mu=\rho\left(c_{s}^{i d}\right)^{2} \Delta t\left[(1+0.5 b \rho g) /\left(\omega^{\prime} g\right)+d /\left(2 A^{2}\right)-1\right]$ and the equation of state being given by

$$
p=\rho R T_{0}(1+b \rho g)=\rho\left(c_{s}^{i d}\right)^{2}(1+b \rho g) .
$$

As compared with the equation of state given by Eq. (9), Eq. (31) includes an additional term to describe the nonideal effect due to the finite size of particles. It follows from Eq. (31) that the density variation with respect to the pressure variation is given by

$$
\frac{\delta \rho}{\delta p}=1 /\left[\left(c_{s}^{i d}\right)^{2} \times\left(1+2 b \rho g+b \rho^{2} g^{\prime}\right)\right]
$$

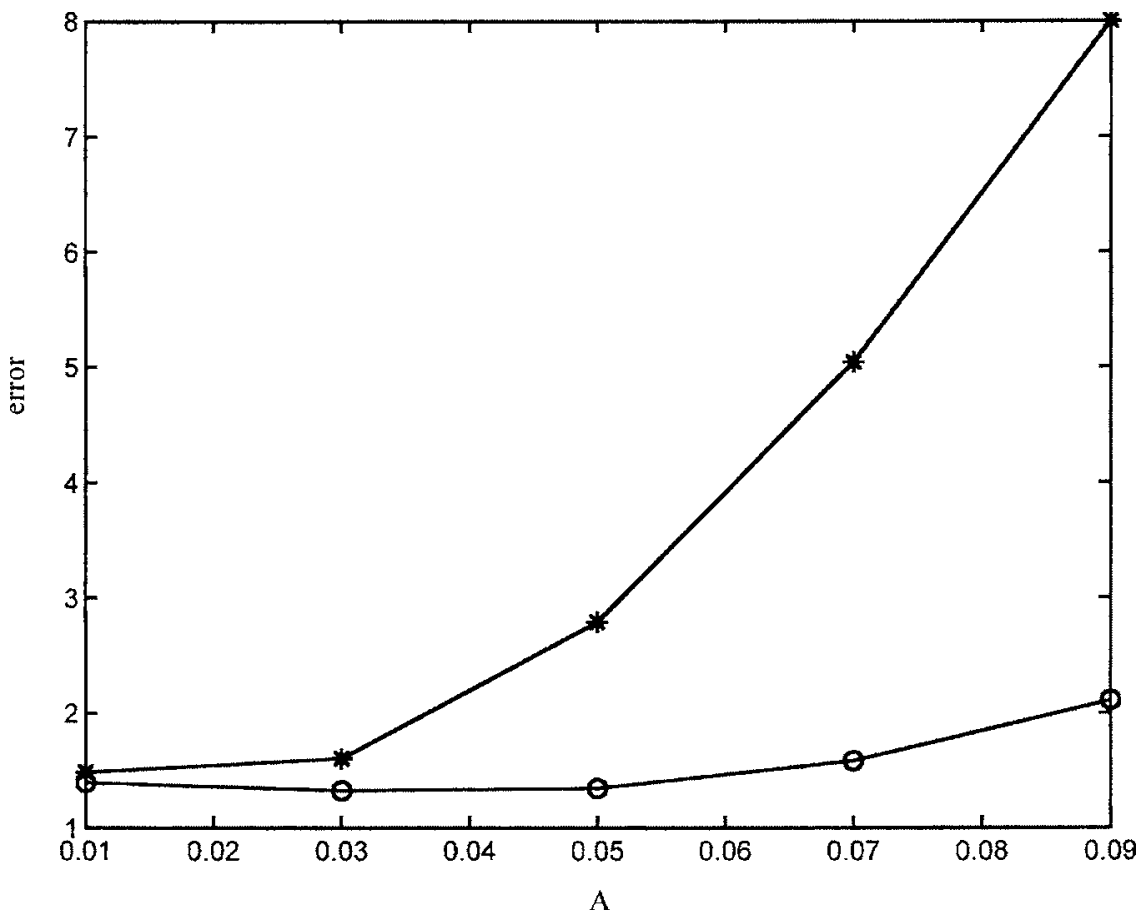

FIG. 6. The average relative error of the center velocity of Womersley flow at $T / 8$ with different amplitude of pressure gradient. - *-: the conventional LBGK model; -O-: the ELBGK model with $b \rho=1.5$. 


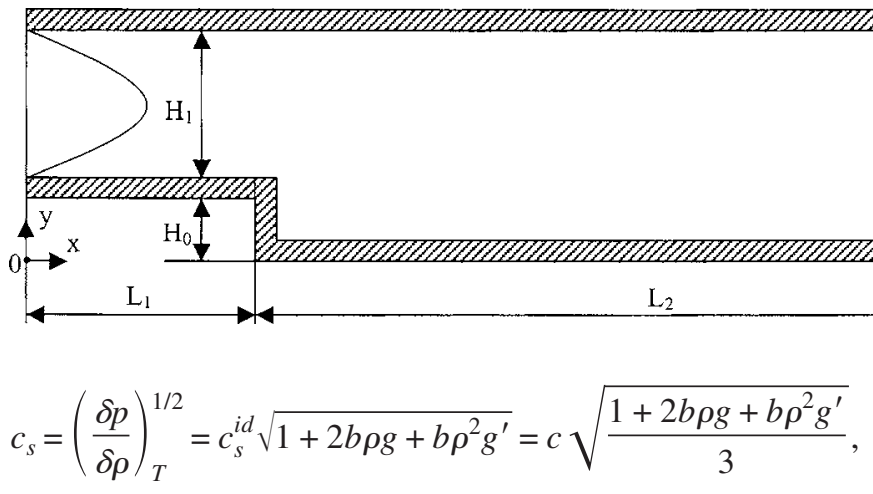

where $g^{\prime}$ denotes $d g / d \rho$. The ratio, $\delta \rho / \delta p$, is not a constant, but depends on $b$ and $\rho$. When $b \rho$ is sufficiently large, the variation of the fluid density becomes small even with a large pressure gradient. Therefore the corresponding compressibility error can be reduced by adjusting the parameter $b \rho$.

\section{NUMERICAL SIMULATION}

In this section, we apply the proposed finite difference ELBGK model to the two dimensional steady Poiseuille flow, and the unsteady Wormersley flow, and the backwardfacing step flow driven by a pressure gradient. The numerical results from the conventional LBGK model for ideal gas (i.e., the D2Q9 model in Ref. [17]) are also presented. To compare the compressibility errors of these two models, we performed all simulations with $c=\sqrt{3 R T_{0}}=1.0$.

\section{A. Poiseuille flow}

Poiseuille flow is driven by a constant pressure gradient $-d p / d x$ along the direction parallel to the walls. Under the steady state, the horizontal velocity is [24]

$$
u=-\frac{L^{2}}{8 \mu} \frac{d p}{d x}\left[1-\left(\frac{y}{L / 2}\right)^{2}\right]
$$

where $L$ is the width between the solid walls, $\mu$ is the fluid viscosity, and $y$ is the distance from the centerline of the channel. We simulated the flow on a $N x \times N y=192 \times 32$ mesh by setting $d p / d x=5 \times 10^{-3}$ and the average fluid density $\bar{\rho}=1.0$. The Reynolds number based on the discrete particle speed $c, \operatorname{Re}_{c}=\bar{\rho} c L / \mu=100$. We applied the pressure (density) boundary conditions [25] to the inlet and outlet, and the nonequilibrium extrapolation method [26] to the top and bottom walls. In the simulations, $J_{i}^{I}$ was discretized by the central difference scheme while

$J_{i}^{I I}$ was discretized by a hybrid of the first order upwind and the central difference schemes:

$$
\begin{gathered}
\left(\frac{\partial \varphi}{\partial x}\right)_{x=x_{i}}=a \frac{\varphi\left(x_{i}\right)-\varphi\left(x_{i-1}\right)}{\Delta x}+(1-a) \frac{\varphi\left(x_{i+1}\right)-\varphi\left(x_{i-1}\right)}{2 \Delta x} \\
\left(c_{i x}>0\right),
\end{gathered}
$$

FIG. 7. The geometry of the backward-facing step flow. with the numerical weight $a=0.05$.

We compare the numerical results obtained by the conventional LBGK model with those by our finite difference ELBGK model with $b \rho$ ranging from 0.5 to 1.5 . Figure 1 shows the velocity profiles at the pressure gradient $d p / d x$ $=5.0 \times 10^{-3}$. It is shown that the velocity profiles given by the different models are almost identical, and all agree well with the analytical solution. This excellent agreement is because such a small pressure gradient causes a rather small density variation. However, it is not the case when the imposed pressure gradient increases. Figure 2 presents the density variation along channel length when the pressure gradient increases up to $d p / d x=2.0 \times 10^{-2}$. It is shown that at this large pressure gradient, the density variation resulting from the conventional LBGK model is one-third of the average density $\bar{\rho}$ and about 15 times larger than that from our finite difference ELBGK model with $b \rho=1.5$. Therefore the velocity profile obtained by the conventional LBGK model deviates significantly from the analytical solution for the incompressible flow. In order to describe this numerical error, we define an average relative error of the velocity as

$$
\text { error }=\frac{1}{N_{y}} \sum_{i} \frac{\left|u\left(y_{i}\right)-\widetilde{u}\left(y_{i}\right)\right|}{\widetilde{u}\left(y_{i}\right)} \times 100 \%,
$$

where $u\left(y_{i}\right)$ and $\tilde{u}$ denote the numerical results and the analytical solution given by Eq. (34), respectively. Figure 3 shows the velocity error of the different models at various pressure gradients. It is shown that when $d p / d x \leqslant 10^{-2}$, the relative errors obtained from the different models are small. Therefore both models are applicable to the flows driven by a small pressure gradient. However, when $d p / d x>10^{-2}$, the relative error of the conventional LBGK model is much larger than that of the finite difference ELBGK model. Taking $d p / d x=2.0 \times 10^{-2}$ as an example, the relative errors of the finite difference ELBGK model with $b \rho=0.5,1.0$, and 1.5 are $1.58,0.7$, and $0.67 \%$ only. At the same pressure gradient, however, the conventional LBGK model yielded the relative error of $8.21 \%$. Thus the conventional LBGK model cannot be applied to simulation of Poiseuille flow with a large pressure gradient.

Figure 3 also indicates that the finite difference ELBGK model becomes unstable when $d p / d x>4.0 \times 10^{2}$ for $b \rho$ $=1.0$ and $d p / d x>2.5 \times 10^{2}$ for $b \rho=1.5$. In fact, this type of numerical instability has also been encountered in other EL- 

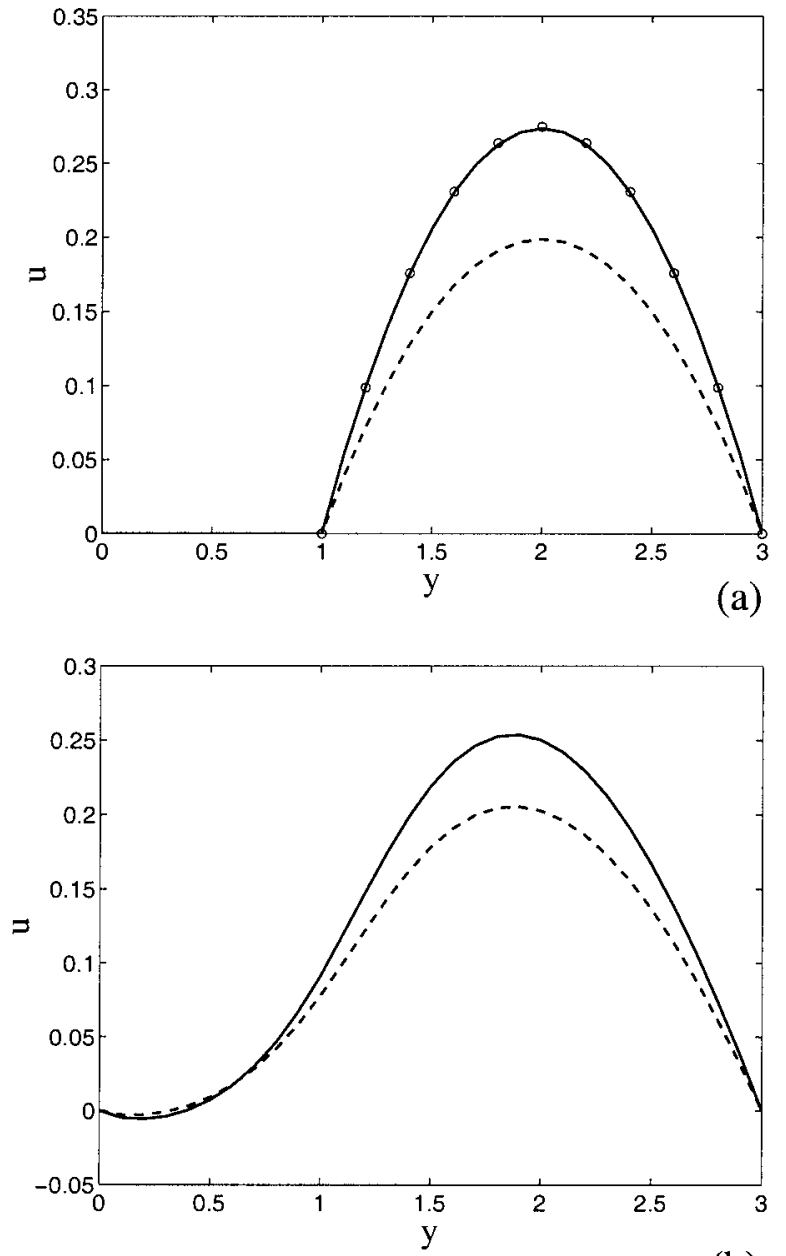

(b)

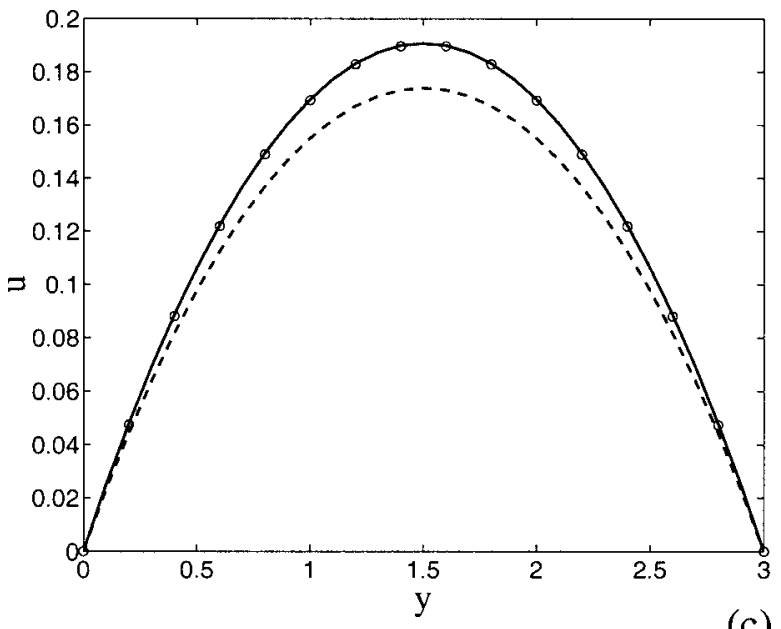

(c)

FIG. 8. The streamwise velocity profiles at (a) $x=0$, (b) $x$ $=5.8 H_{0}$, (c) $x=21 H_{0}$ in the backward-facing step flow with $\left(p_{\text {in }}\right.$ $\left.-p_{\text {out }}\right) /\left(L_{1}+L_{2}\right)=5.0 \times 10^{-3}$. Solid line: the ELBGK model with $b \rho=1.0$; dashed line: the conventional LBGK model; - $\bigcirc-$ : analytical incompressible solution.

BGK models [20-23]. This is because the evolution equation of the ELBGK models includes the terms of gradients, i.e., $J_{i}^{I}$ and $J_{i}^{I I}$. However, it is worth mentioning that for a given $b \rho$,

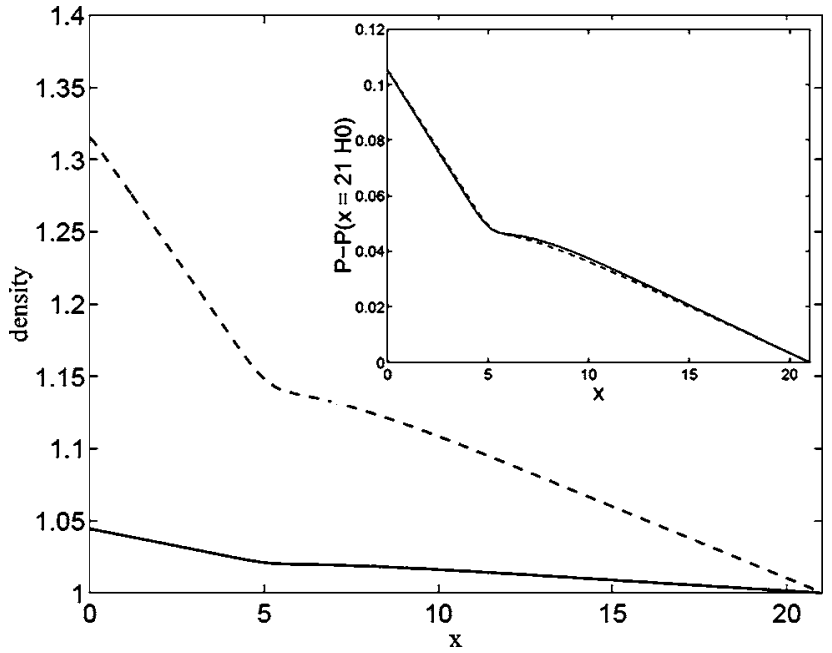

FIG. 9. The density variation along the channel in the backwardfacing step flow with $\left(p_{\text {in }}-p_{\text {out }}\right) /\left(L_{1}+L_{2}\right)=5.0 \times 10^{-3}$. Solid line: the ELBGK model with $b \rho=1.0$; dashed line: the conventional LBGK model. Inset: the pressure variation along the channel in the backward facing step flow with $\left(p_{\text {in }}-p_{\text {out }}\right) /\left(L_{1}+L_{2}\right)=5.0 \times 10^{-3}$. Solid line: the ELBGK model with $b \rho=1.0$; dashed line: the conventional LBGK model.

the ELBGK model proposed in this work can remain stable in a much wider pressure gradient range than can other ELBGK models [20-23]. Such an improvement in stability is due to the increased hyperviscosity as a result of utilizing the finite difference scheme [27]. Figure 3 also shows that the conventional LBGK model will become unstable when $d p / d x>5.0 \times 10^{2}$, which, however, is caused by the large density variation as a result of the large pressure gradient.

\section{B. Womersley flow}

We now present the numerical results obtained by the finite difference ELBGK model for the unsteady Womersley flow. Womersley flow is driven by a periodic pressure gradient:

$$
\frac{d p}{d x}=A_{p} \cos (\omega t)=\operatorname{Re}\left(A_{p} e^{i \omega t}\right)
$$

where $A_{p}$ is the amplitude and $\omega$ is frequency. If the flow is laminar, the corresponding governing equation is

$$
\frac{\partial u}{\partial t}=-\frac{1}{\rho} \frac{\partial p}{\partial x}+\nu \frac{\partial^{2} u}{\partial y^{2}}
$$

$$
\nu=0,
$$

where $u$, and $\nu$ are, respectively, the components of the velocity parallel and perpendicular to the walls. Equations (38) and (39) can be solved analytically to give [28]

$$
u=\operatorname{Re}\left[i \frac{A_{p}}{\omega}\left(1-\frac{\cos \left[\lambda\left(2.0 \times y^{*}-1\right)\right]}{\cos \lambda}\right) e^{i \omega t}\right],
$$

where $y^{*}=y / L$, with $L$ being the channel width, and $\lambda$ $=\sqrt{-i W o^{2}}$, with the Womersley number $\mathrm{Wo}^{2}=L^{2} \omega /(4 \gamma)$. We 
performed simulation of the flow on a $N x \times N y=192 \times 32$ grid and employed the same boundary treatments as those used in Poiseuille flow. The amplitude and period of the pressure drop are set to be $A_{p}=1.0^{-2}$ and $T=2 \pi / \omega=25$, respectively. In our simulation, the average density of the whole flow field $\bar{\rho}$ was set to 1.0 and the Womersley number Wo $=2.0$. We commenced the simulation at $(u, v)=(0,0)$ and measured the oscillating velocity field after $5 T$. Figure 4 compares the velocity profiles obtained by the conventional LBGK model with those obtained by the finite difference ELBKG model with $b \rho=1.5$ at $T / 8, T / 4,3 T / 8$, and $T / 2$ when $A_{p}=1.0^{-2}$. It is evident that in the case of the flow driven by a small periodic pressure gradient, the velocities obtained by the two different models are almost identical. This reflects under the circumstance of small pressure gradient, the compressibility error has negligible impact on the velocity field. However, this is not the case when the amplitude of the pressure gradient becomes larger. Figure 5 shows the velocity profiles for $A_{p}=7.0 \times 10^{-2}$. It is evident that the velocity profile obtained by the conventional LBGK model deviates significantly from the incompressible analytical solution, while the finite difference ELBGK model with $b \rho$ $=1.5$ still predicts the flow well. Figure 6 further shows the corresponding average center velocity error at $t=T / 8$ with the amplitude of pressure gradient from $1.0^{-2}$ to 9.0 $\times 1.0^{-2}$. The error of the conventional LBGK model increases dramatically. Taking the case of $A_{p}=7.0 \times 1.0^{-2}$ as an example, the error of the conventional LBGK model is about $5.04 \%$ while the error of the finite difference ELBGK model with $b \rho=1.5$ is $1.58 \%$ only. This validation shows that the finite difference ELBGK model proposed in this work can effectively suppress the compressibility error for unsteady flows with a large pressure gradient.

\section{Backward-facing step flow}

We also validated our finite difference ELBGK model by simulating the backward-facing step flow, in which, unlike Poiseuille flow and Womersley flow presented above, the nonlinear advection term is included.

The geometric configuration of the backward-facing step flow is sketched in Fig. 7, where $H_{0}$ is the height of the backward facing step, $L_{1}$ and $L_{2}$ are the length of the channel before the step and the channel after the step, respectively. In our simulations, we set $H_{0}=1.0, L_{1}=5 H_{0}$, and $L_{2}=16 H_{0}$. Moreover, we chose the height of the channel before the step $H_{1}=2 H_{0}$ and the height of the channel after the step $\mathrm{H}_{2}$ $=3 H_{0}$, thereby the characteristic expansion ratio $H_{2} / H_{1}$ is equal to $3 / 2$. The Reynolds number is based on the discrete particle speed $c, \operatorname{Re}=c H_{0} / \nu=50$. We simulated the flow on a $N x \times N y=210 \times 30$ mesh and applied the same boundary condition treatments as those in Poiseuille flow.

We found that when the pressure gradient along the channel is very small, the numerical results obtained by the conventional LBGK model agreed well with those simulated by our finite difference ELBGK model. However, when the pressure gradient increases, our model predicts more accurate results. Figure 8 presents the streamwise velocity profiles at different locations along the channel obtained by the conventional LBGK model and our model with $b \rho=1.0$ when $\left(p_{\text {in }}-p_{\text {out }}\right) /\left(L_{1}+L_{2}\right)=5.0 \times 10^{-3}$. It shows that the streamwise velocity predicted by the conventional LBGK model is always much smaller than that by our finite difference ELBGK model. We obtained the numerical solutions of the fully developed velocity profiles at the inlet of the channel before the step and the outlet of the channel after the step, and compared them with the analytical solution in Fig. 8 . It is seen that the velocity profiles obtained by the finite difference ELBGK model with $b \rho=1.0$ well predicts the flow, but the conventional LBGK model deviates significantly from the analytical solution.

Figure 9 compares the resulting densities at the centerline of the channel $\rho\left(y=2 H_{0}\right)$, obtained by the two models. It is seen that with the same pressure difference shown in the inset in Fig. 9, the density resulting from the conventional LBGK model exhibits a much larger change than that from our model. Therefore this pronounced density variation indicates that the conventional LBGK model creates a large compressibility error in simulating this large pressure gradient flow. On the other hand, the rather small density variation resulting from our model implies that the compressibility error is significantly reduced. The above presented numerical results indicate that the finite difference ELBGK model developed in this work is applicable to nonlinear incompressible flows with a large pressure gradient.

\section{CONCLUSION}

Conventional LBGK models developed from the Boltzmann equation result in the equation of states of ideal gas. When they are applied to simulation of incompressible flows with a large pressure gradient, a large compressibility error will arise and lead to the numerical results deviating significantly from the real solutions. In this work, we developed a lattice BGK model based on the Enskog equation. Unlike the conventional LBGK models, the transport properties and the equation of state resulting from this ELBGK model are those of nonideal fluids. We discretized this ELBGK model and applied the resulting finite difference numerical scheme to simulation of the two dimensional Poiseuille flow, Womersley flow, and backward-facing step flow. It has been shown that the finite difference ELBGK model can predict the flows with much more accurate solutions than do the conventional LBGK models.

\section{ACKNOWLEDGMENT}

The work described in this paper was fully supported by a grant from the Research Grants Council of the Hong Kong Special Administrative Region, China (Project No. HKUST6101/04E).

\section{APPENDIX: RECOVERING THE NAVIER-STOKES EQUATIONS FROM THE FINITE DIFFERENCE ENSKOG-EQUATION BASED LATTICE BGK MODEL}

In this section, we will recover the Navier-Stokes equations from our finite difference Enskog-equation based lattice 
BGK model. First we rewrite its evolution equation,

$$
h_{i}^{+}(t, \vec{r})=h_{i}(t, \vec{r})-g \omega^{\prime}\left[h_{i}(t, \vec{r})-h_{i}^{e q}(t, \vec{r})\right]+\Delta t\left(J_{i}^{I}+J_{i}^{I I}\right) ;
$$

and

$$
\begin{aligned}
h_{i}(t+\Delta t, \vec{r})= & h_{i}^{+}(t, \vec{r})+\frac{d}{2}\left[h_{i}^{+}\left(t, \vec{r}+\vec{c}_{i} \Delta t / A\right)-2 h_{i}^{+}(t, \vec{r})\right. \\
& \left.+h_{i}^{+}\left(t, \vec{r}-\vec{c}_{i} \Delta t / A\right)\right]-\frac{A}{2}\left[h_{i}^{+}\left(t, \vec{r}+\vec{c}_{i} \Delta t / A\right)\right. \\
& \left.-h_{i}^{+}\left(t, \vec{r}-\vec{c}_{i} \Delta t / A\right)\right] .
\end{aligned}
$$

By expanding $h_{i}(t+\Delta t, \vec{r}), \quad h_{i}^{+}\left(t, \vec{r}+\vec{c}_{i} \Delta t / A\right)$, and $h_{i}^{+}(t, \vec{r}$ $\left.-\vec{c}_{i} \Delta t / A\right)$ in a Taylor series about $\vec{r}, t$, Eq. (A2) can be approximated by

$$
\begin{aligned}
h_{i}+\Delta t \frac{\partial h_{i}}{\partial t}+\frac{\Delta t^{2}}{2} \frac{\partial^{2} h_{i}}{\partial t^{2}}= & h_{i}^{+}(t, \vec{r})+\frac{d \Delta t^{2}}{2 A^{2}}\left[\vec{c}_{i} \vec{c}_{i}: \frac{\partial}{\partial \vec{r}}\left(\frac{\partial h_{i}^{+}}{\partial \vec{r}}\right)\right] \\
& -\vec{c}_{i} \Delta t \cdot \frac{\partial h_{i}^{+}}{\partial \vec{r}}+O\left(\Delta t^{3}\right) .
\end{aligned}
$$

We now introduce the Chapman-Enskog multiscale expansion,

$$
\begin{gathered}
\frac{\partial}{\partial t}=K n \frac{\partial_{1}}{\partial t}+K n^{2} \frac{\partial_{2}}{\partial t}, \\
\frac{\partial}{\partial \vec{r}}=K n \frac{\partial_{1}}{\partial \vec{r}}, \\
h_{i}=\sum_{n=0}^{\infty} K n^{n} h_{i}^{(n)}=\sum_{n=0}^{\infty} K n^{n} f_{i}^{(n)}-K n \frac{\Delta t}{2} J_{i, 1}^{I},
\end{gathered}
$$

where $J_{i, 1}^{I}=-f_{i}^{e q} b \rho g\left(\vec{c}_{i}-\vec{u}\right) \cdot \partial_{1} \ln \left(\rho^{2} g\right) / \partial \vec{r}$. With Eqs. (A1), (A4), (A5), and (A6), Eq. (A3) can be expanded into a series of equations in different order of $\mathrm{Kn}$,

$$
\begin{gathered}
K n^{0}: \quad h_{i}^{(0)}=f_{i}^{(0)}=f_{i}^{(e q)}, \\
K n^{1}: \quad \frac{\partial_{1} h_{i}^{(0)}}{\partial t}+\vec{c}_{i} \cdot \frac{\partial_{1} h_{i}^{(0)}}{\partial \vec{r}}=-\frac{g}{\tau^{\prime}} f_{i}^{(1)}+J_{i, 1}^{I}, \\
K n^{2}: \quad \frac{\partial_{2} h_{i}^{(0)}}{\partial t}+\frac{\partial_{1} h_{i}^{(1)}}{\partial t}+\vec{c}_{i} \cdot \frac{\partial_{1} h_{i}^{(1)}}{\partial \vec{r}} \\
=-\frac{g}{\tau^{\prime}} h_{i}^{(2)}+J_{i, 1}^{I I}+\frac{d \Delta t}{2 A^{2}}\left[\vec{c}_{i} \vec{c}_{i}: \frac{\partial_{1}}{\partial \vec{r}}\left(\frac{\partial_{1} h_{i}^{(0)}}{\partial \vec{r}}\right)\right]-\frac{\Delta t}{2} \frac{\partial_{1}^{2} h_{i}^{(0)}}{\partial t^{2}} \\
-\vec{c}_{i} \Delta t \cdot \frac{\partial_{1}\left(-g / \tau^{\prime} f_{i}^{(1)}+J_{i, 1}^{I}\right)}{\partial \vec{r}},
\end{gathered}
$$

where $\quad h_{i}^{(1)}=f_{i}^{(1)}-\Delta t / 2 J_{i, 1}^{I}, \quad h_{i}^{(2)}=f_{i}^{(2)}, \quad$ and $J_{i, 1}^{I I}=-0.5 \vec{c}_{i} \cdot \partial_{1}\left(f_{i}^{(1)} b \rho g\right) / \partial \vec{r}$. With the help of Eq. (A8), Eq. (A9) can be further reduced to

$$
\begin{gathered}
\frac{\partial_{2} h_{i}^{(0)}}{\partial t}+\left(1-\frac{g \Delta t}{2 \tau^{\prime}}\right)\left(\frac{\partial_{1} f_{i}^{(1)}}{\partial t}+\vec{c}_{i} \cdot \frac{\partial_{1} f_{i}^{(1)}}{\partial \vec{r}}\right)+\frac{\Delta t}{2}\left(1-\frac{d}{A^{2}}\right) \\
\times\left[\vec{c}_{i} \vec{c}_{i}: \frac{\partial_{1}}{\partial \vec{r}}\left(\frac{\partial_{1} h_{i}^{(0)}}{\partial \vec{r}}\right)\right]=-\frac{g}{\tau^{\prime}} h_{i}^{(2)}+J_{i, 1}^{I I} .
\end{gathered}
$$

From Eqs. (A7) and (23) and the definition of $f_{i}^{e q}$ given by Eq. (3), we have the velocity moments in different order of $J_{i}^{I}, f_{i}^{(n)}$, s, and $h_{i}^{(n)}$, s,

$$
\begin{gathered}
\sum_{i=0}^{9} m f_{i}^{(0)}=\rho, \quad \sum_{i=0}^{9} m f_{i}^{(n)}=0 \quad \text { for }(n>0), \\
\sum_{i=0}^{9} m \vec{c}_{i} f_{i}^{(0)}=\rho \vec{u}, \quad \sum_{i=0}^{9} m \vec{c}_{i} f_{i}^{(n)}=0 \quad \text { for }(n>0),
\end{gathered}
$$

$$
\begin{gathered}
\sum_{i=0}^{9} m J_{i}^{I}=0, \quad \sum_{i=0}^{9} m \vec{c}_{i} J_{i}^{I}=-b\left(c_{s}^{i d}\right)^{2} \frac{\partial}{\partial \vec{r}}\left(\rho^{2} g\right), \\
\sum_{i=0}^{9} m \vec{c}_{i} \vec{c}_{i} J_{i}^{I}=b\left\{\vec{u} \vec{u} \vec{u} \cdot \frac{\partial}{\partial \vec{r}}\left(\rho^{2} g\right)-\left(c_{s}^{i d}\right)^{2}\left[\left(\vec{u} \frac{\partial}{\partial \vec{r}}\left(\rho^{2} g\right)\right)\right.\right. \\
\left.\left.+\left(\vec{u} \frac{\partial}{\partial \vec{r}}\left(\rho^{2} g\right)\right)^{T}\right]\right\}, \\
\sum_{i=0}^{9} m h_{i}^{(0)}=\rho, \quad \sum_{i=0}^{9} m h_{i}^{(n)}=0 \quad \text { for }(n>0), \\
\sum_{i=0}^{9} m \vec{c}_{i} h_{i}^{(0)}=\rho \vec{u}, \\
\sum_{i=0}^{9} m \vec{c}_{i} \vec{c}_{i} h_{i}^{(0)}=\rho \vec{u} \vec{u}+\rho\left(c_{s}^{i d}\right)^{2} \overrightarrow{\vec{I}}, \\
\sum_{i=0}^{9} m \vec{c}_{i} h_{i}^{(1)}=\frac{\Delta t}{2} b\left(c_{s}^{i d}\right)^{2} \frac{\partial}{\partial \vec{r}}\left(\rho^{2} g\right), \\
\sum_{i=0}^{9} m \vec{c}_{i} h_{i}^{(n)}=0 \quad \text { for }(n>1),
\end{gathered}
$$

where $\overrightarrow{\vec{I}}$ is the second order unit tensor, superscript $T$ denotes the transpose. With the help of Eqs. (A11)-(A15), we can recover the Euler equation from Eq. (A8):

$$
\begin{gathered}
\frac{\partial_{1} \rho}{\partial t}+\frac{\partial_{1}}{\partial \vec{r}} \cdot(\rho \vec{u})=0, \\
\frac{\partial_{1} \vec{u}}{\partial t}+\vec{u} \cdot \frac{\partial_{1}}{\partial \vec{r}}(\vec{u})=-\frac{1}{\rho} \frac{\partial_{1}}{\partial \vec{r}} p,
\end{gathered}
$$

with $p=\rho\left(c_{s}^{i d}\right)^{2}(1+b \rho g)$. 
On the other hand, from Eq. (A8), we have

$$
f_{i}^{(1)}=\left(-\frac{\tau^{\prime}}{g}\right)\left(\frac{\partial_{1} h_{i}^{(0)}}{\partial t}+\vec{c}_{i} \cdot \frac{\partial_{1} h_{i}^{(0)}}{\partial \vec{r}}-J_{i, 1}^{I}\right) .
$$

Therefore, with the aid of Eqs. (A13), (A17), and (A18), the second order velocity moment of $f_{i}^{(1)}$ is

$$
\sum_{i=1}^{9} c_{i \alpha} c_{i \beta} f_{i}^{(1)}=\left(-\frac{\tau^{\prime}}{g}\right) \rho\left(c_{s}^{i d}\right)^{2} \frac{\partial_{1}}{\partial \vec{r}}(\vec{u})+O\left(M^{2}\right) .
$$

Now multiplying $\psi=\{m, m \vec{c}\}$ on both sides of Eq. (A10), and summing up all discrete particle velocity, with the help of Eqs. (A11)-(A16) and (A20), we get the corresponding macroscopic equations in order of $K n^{2}$ :

$$
\frac{\partial_{2} \rho}{\partial t}=O\left(\Delta t M^{2}\right)
$$

$$
\frac{\rho \partial_{2} \vec{u}}{\partial t}-\frac{\partial_{1}}{\partial \vec{r}} \cdot\left(\mu \frac{\partial_{1}}{\partial \vec{r}}(\vec{u})\right)=O\left(M^{2}\right),
$$

where the viscosity $\mu=\rho\left(c_{s}^{i d}\right)^{2} \Delta t\left[(1+0.5 b \rho g) /\left(\omega^{\prime} g\right)\right.$ $\left.\left.+d / 2 A^{2}\right)-1\right]$. In conclusion, in the incompressible limit $(M$ $\ll 1$ ), combining Eqs. (A17) and (A21) and Eqs. (A18) and (A22), we can obtain the macroscopic mass and momentum conservation equation:

$$
\begin{gathered}
\frac{\partial \rho}{\partial t}+\frac{\partial}{\partial \vec{r}} \cdot(\rho \vec{u})=0 \\
\rho\left[\frac{\partial \vec{u}}{\partial t}+\vec{u} \cdot \frac{\partial}{\partial \vec{u}}(\vec{u})\right]=-\frac{\partial}{\partial \vec{r}} p+\frac{\partial}{\partial \vec{r}} \cdot\left(\mu \frac{\partial}{\partial \vec{r}}(\vec{u})\right) .
\end{gathered}
$$

[1] Y. H. Qian, S. Succi, and S. A. Orszag, Annu. Rev. Comput. Phys. 3, 195 (1995).

[2] S. Chen and G. D. Doolen, Annu. Rev. Fluid Mech. 30, 329 (1998).

[3] D. A. Wolf-Gladrow, Lattice-gas Cellular Automata and Lattice Boltzmann Models: An Introduction (Springer-Verlag, Berlin, 2000).

[4] S. Succi, The Lattice Boltzmann Equation for Fluid Dynamics and Beyond (Oxford University Press, New York, 2001).

[5] D. Z. Yu, R. W. Mei, L. S. Luo, and S. Wei, Prog. Aerosp. Sci. 39, 329 (2003).

[6] Z. Lin, H. Fang, and R. Tao, Phys. Rev. E 54, 6323 (1996).

[7] Y. Chen and H. Ohashi, Int. J. Mod. Phys. C 8, 793 (1997).

[8] Q. Zou, S. Hou, S. Chen, and G. D. Doolen, J. Stat. Phys. 81, 35 (1995).

[9] X. He and L. S. Luo, J. Stat. Phys. 88, 927 (1997).

[10] Z. L. Guo, B. Shi, and N. Wang, J. Comput. Phys. 165, 288 (2000).

[11] H. P. Fang, R. Z. Wan, and Z. F. Lin, Phys. Rev. E 66, 036314 (2002).

[12] S. Marconi, B. Chopard, and J. Latt, Int. J. Mod. Phys. C 14, 1015 (2003).

[13] S. Chapman and T. G. Cowling, The Mathematical Theory of Non-uniform Gases: An Account of the Kinetic Theory of Viscosity, Thermal Conduction and Diffusion in Gases (Cambridge University Press, Cambridge, UK, 1990).
[14] J. O. Hirschfelder, C. F. Curtiss, and R. B. Bird, Molecular Theory of Gases and Liquids (John Wiley \& Sons, New York, 1964).

[15] T. M. Reed and K. E. Gubbins, Applied Statistical Mechanics: Thermodynamic and Transport Properties of Fluids (Butterworth-Heinemann, Boston, 1991).

[16] Z. L. Guo, C. Zheng, and T. S. Zhao, J. Sci. Comput. 16, 569 (2001).

[17] Y. H. Qian, D. D’Humières, and P. Lallemand, Europhys. Lett. 17, 479 (1992).

[18] X. He and L. S. Luo, Phys. Rev. E 55, R6333 (1997).

[19] X. He and L. S. Luo, Phys. Rev. E 56, 6811 (1997).

[20] L. S. Luo, Phys. Rev. E 62, 4982 (2000).

[21] L. S. Luo, Phys. Rev. Lett. 81, 1618 (1998).

[22] X. He and G. D. Doolen, J. Stat. Phys. 107, 309 (2002).

[23] X. He, X. Shan, and G. D. Doolen, Phys. Rev. E 57, R13 (1998).

[24] A. Bejan, Convection Heat Transfer (John Wiley \& Sons, New York, 1984).

[25] Z. L. Guo, C. G. Zheng, and B. C. Shi, Chin. Phys. 11, 366 (2002).

[26] Z. L. Guo, C. G. Zheng, and B. C. Shi, Phys. Fluids 14, 2007 (2002).

[27] P. Lallemand and L. S. Luo, Phys. Rev. E 68, 036706 (2003).

[28] I. G. Currie, Fundamental Mechanics of Fluids (Marcel Dekker, New York, 2003). 